\title{
Thermal properties of slow dynamics ${ }^{1}$
}

\author{
Leticia Cugliandolo $^{\text {a,2 }} \underline{\text { Jorge Kurchan }}^{\text {b,3 }}$ \\ ${ }^{a}$ Laboratoire de Physique Théorique de l'École Normale Supérieure, \\ 24 rue Lhomond, 75231 Paris Cedex 05, France \\ and \\ Laboratoire de Physique Théorique et Hautes Energies, Jussieu \\ 5 ème étage, Tour 24, 4 Place Jussieu, 75005 Paris France \\ ${ }^{\mathrm{b}}$ Laboratoire de Physique Théorique de l' Ecole Normale Supérieure de Lyon \\ 46 Allée d'Italie, Lyon, France
}

\begin{abstract}
The limit of small entropy production is reached in relaxing systems long after preparation, and in stationary driven systems in the limit of small driving power. Surprisingly, for extended systems this limit is not in general the Gibbs-Boltzmann distribution, or a small departure from it. Interesting cases in which it is not are glasses, phase-separation, and certain driven complex fluids.

We describe a scenario with several coexisting temperatures acting on different timescales, and partial equilibrations at each time scale. This scenario entails strong modifications of the fluctuation-dissipation equalities and the existence of some unexpected reciprocity relations. Both predictions are open to experimental verification, particularly the latter.

The construction is consistent in general, since it can be viewed as the breaking of a symmetry down to a residual group. It does not assume the presence of quenched disorder. It can be - and to a certain extent has been - tested numerically, while some experiments are on their way. There is furthermore the perspective that analytic arguments may be constructed to prove or disprove its generality.
\end{abstract}

Key words: Nonequilibrium extensive systems.

Pacs numbers: 75.40.Gb, 75.10.Nr, 02.50.-r, 05.20.-y

1 Invited talk to be presented at STATPHYS 20, Paris.

Paper dedicated to Heinz Horner on occasion of his $60^{\text {th }}$ birthday.

2 leticia@physique.ens.fr

3 jkurchan@enslapp.ens-lyon.fr

Preprint submitted to Elsevier Preprint

7 July 2021 


\section{Slow dynamics}

\subsection{Two complementary aspects: driven and aging systems.}

A universal feature of systems with slow dynamics is the extreme sensitivity of their time-dependencies to perturbations. Glassy systems which 'age' if left to relax (the correlations evolve slower and slower as time passes) may become stationary if an even small amount of power is pumped into them. Thus, for example, a 3D spin-glass having the characteristic aging autocorrelation curves (Fig. $3 a$ ) ), becomes stationary upon applying a very weak, slowly evolving random field (Fig. $3 \mathrm{c}$ ) ). The reason for this sensitivity is the presence of flat directions in phase-space, indeed the very origin of the slowness of the dynamics.

Remarkably, some properties remain unaltered even when the time-dependence has been dramatically changed by a small perturbation. Precisely these properties are universal in slow systems, at least qualitatively.

What this suggests is that we treat on an equal footing all problems with slow dynamics, whether they age or are stationary [1]. Their relevant common feature [4] (indeed, the definition of 'slow') is the smallness of the quantity that can be identified as an entropy production [5] in each case. Thus, we refer collectively as the 'small entropy production' (SEP) limit to the situation reached at long times and/or small external power input.

\subsection{Fluctuation-dissipation temperatures.}

In the SEP limit many systems are close to equilibrium. Such situation has been well studied in the past (see e.g. [5]). However, this is not the generic situation, particularly in complex systems such as glasses, phase-separating fluids, moving objects that are just unpinning, etc. An example will at this point clarify things. Consider a system with variables $\phi_{i}(t)$, their correlation functions $C_{i j}\left(t, t^{\prime}\right)=\left\langle\phi_{i}(t) \phi_{j}\left(t^{\prime}\right)\right\rangle$, and the linear response $R_{i j}\left(t, t^{\prime}\right)=\left.\frac{\delta\left\langle\phi_{i}(t)\right\rangle}{\delta h_{j}\left(t^{\prime}\right)}\right|_{h=0}$ of $\phi_{i}$ to a kick applied to $\phi_{j}$. If we make a parametric plot of the integrated response

$$
\chi_{i j}\left(t+t_{w}, t_{w}\right)=\int_{t_{w}}^{t+t_{w}} d t^{\prime} R_{i j}\left(t+t_{w}, t^{\prime}\right)
$$

versus the corresponding correlation $C_{i j}\left(t+t_{w}, t_{w}\right)$, the fluctuation-dissipation theorem is the statement that the plot gives a straight line of gradient $-\beta=$ 


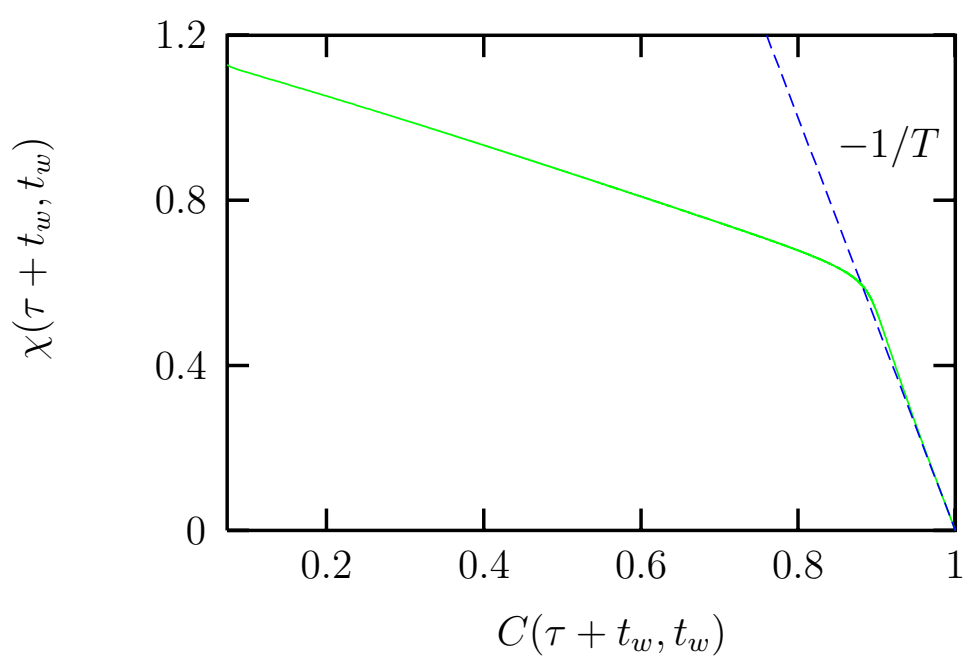

Fig. 1. Integrated response versus correlation plot for a mean-field glass model.

$-1 / T$. For an aging system we get instead, for large $t_{w}$, a limit curve that looks qualitatively like Fig. 1. This figure has been obtained for the autocorrelation of the variables in a mean-field glass model [13], but similar plots have been obtained numerically for 3 and 4 dimensional spin-glasses [6], Lennard-Jones glasses with Montecarlo [7] and molecular dynamics [8]. Ordinary domain growth (as well as droplet models for spin-glasses) [9] also yield such a curve, with the peculiarity that the line to the left becomes horizontal.

These systems age. As mentioned above, we can perturb them with small, nonconservative forces which render them stationary. Remarkably, at least in every model we know, if we now make the plots of Fig. 1 we get, even for small driving powers, the same dependence [2,3]. Despite the fact that the driven systems are stationary, they are well out of equilibrium.

Such robustness of the plot in Fig. 1 suggests that the fluctuation-dissipation ratio might have a physical meaning. Let us define, for any two observables and for any two times, the effective temperatures as [10]

$$
\beta_{i j}^{e f f}\left(\omega, t_{w}\right) \equiv \frac{\chi_{i j}^{\prime \prime}\left(\omega, t_{w}\right)}{\omega \operatorname{Re} \tilde{C}_{i j}\left(\omega, t_{w}\right)}
$$

We have Fourier transformed the time-difference, as in experiments. In a stationary system the $t_{w}$ dependence dissappears.

\subsection{Scales}

Equation (2) is just a definition. The scenario we shall discuss here consists of saying that for all pairs of observables and for each timescale there is a single 
effective temperature. That is,

$$
\beta_{i j}^{e f f}\left(\omega, t_{w}\right)-\beta_{k l}^{e f f}\left(\omega, t_{w}\right) \sim 0 \quad \text { or } \quad \beta_{i j}^{e f f}\left(\omega, t_{w}\right) \sim \beta^{e f f}\left(\omega, t_{w}\right), \quad \forall i, j
$$

and

$$
\beta^{e f f}\left(\omega_{1}, t_{w}\right)-\beta^{e f f}\left(\omega_{2}, t_{w}\right) \sim 0 \quad \text { if } \frac{\omega_{1}}{\omega_{2}}=\text { const. }
$$

in the SEP limit $\left(t_{w} \rightarrow \infty\right.$ and vanishing driving power). A system with a $\chi$ vs. $C$ plot like Fig. 1 (two straight lines) then has two temperatures (one of them being $T$ ). In systems with more than two temperatures the plot has a straight line of gradient $-1 / T$ plus a piece of non-constant slope.

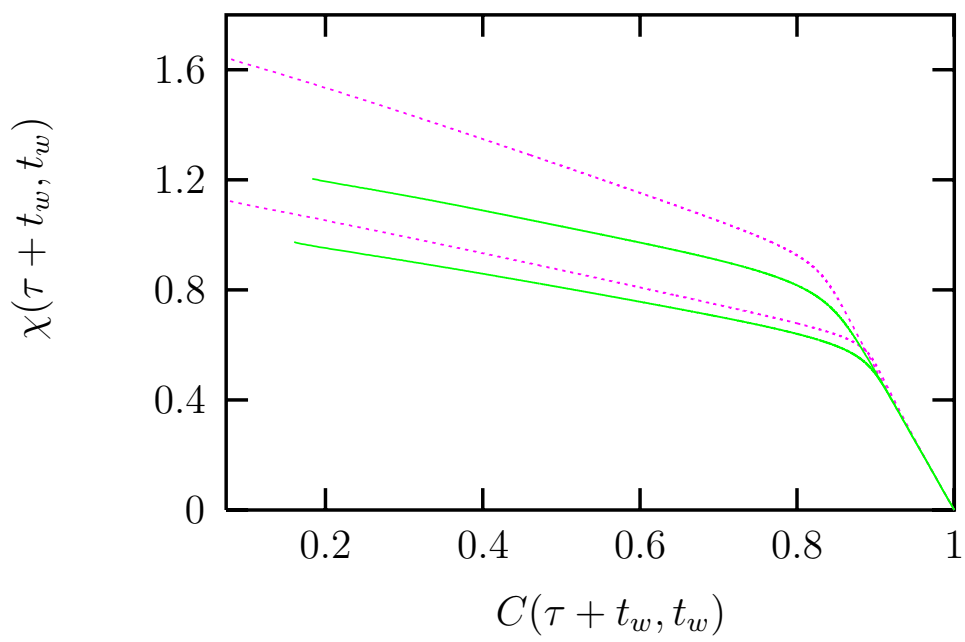

Fig. 2. Thermalisation of two subsystems. Integrated response vs. correlation plots for two uncoupled systems (dots) and for systems that are coupled (lines).

\subsection{Thermometry. Zeroth law.}

One can now ask if (2) really defines temperatures. To check this, one couples a 'thermometer' system simultaneously to the same observable of an ensemble of many independent copies of the system [10,11]. One can then show that the copies of the system act on the thermometer as a superposition of thermal baths with the corresponding effective temperatures and timescales. In order to measure a single temperature, one can construct a tuned thermometer (e.g. an oscillator) that responds to a single time-scale.

To see the relation of FDT-temperatures with the zeroth law, one can couple the 'thermometer' as before, but alternatively to two observables. Then one shows that there is on average a net heat flow from the higher to the lower effective temperature. 


\subsection{Thermalisation of subsystems}

Within the present scenario, two systems with different effective temperatures if weakly coupled eventually 'thermalise' as follows: If the coupling is relatively strong [10], we have the situation of Fig. 2 (a direct solution of the equations, without assumptions): two (mean-field) glassy systems were defined so as to have different effective temperatures when uncoupled (dotted lines). If they are made to evolve coupled, the effective temperatures equalise (full lines).

If the coupling is weak [11], the system preserves essentially the temperatures of its constituents, but eventually rearranges them in widely separated timescales. Thus, the combined system has three temperatures, the bath temperature plus other two, each acting on a separate timescale.

\subsection{Auxiliary thermal baths}

An interesting tool for the study of a system with more than one 'natural' temperature is to couple it to a slow, weak auxiliary bath [11] of temperature $T^{*}$, which, as we shall see, plays the role of 'a field conjugate to the natural temperatures' [12]. The outcome, within this scenario, is that the auxiliary bath fixes the timescale at which its temperature happens in the system. In particular, an aging system with a single $T^{e f f}>T$ will become stationary if $T^{*}>T_{\text {eff }}$, and be hardly affected if $T^{*}<T^{e f f}$. More generally, an aging system with multiple effective temperatures should become partially stationary (for all the timescales with $T^{*}>T^{e f f}$ ), but would still have aging for timescales with $T^{*}<T^{e f f}$. Simulations for the three dimensional EdwardsAnderson model (a system which seems to have many temperatures [6]) are in this sense very encouraging (see Fig. 3).

\subsection{Reciprocity}

A surprising feature of the present scenario is that in the SEP limit there are reciprocity relations $\left\langle A(t) B\left(t^{\prime}\right)\right\rangle=\left\langle B(t) A\left(t^{\prime}\right)\right\rangle$, and similarly for the responses.

It is remarkable that reciprocity relations hold in a situation in which FDT is strongly violated. This is all the more surprising in an aging case in which the system is not even stationary. The interest of these relations is that they are relatively easy to measure in a simulation or in an experiment.

Figure 4 shows a numerical check for two $3 \mathrm{D}$ spin glasses. $C_{11}$ and $C_{22}$ denote the autocorrelations of systems 1 and 2 (which have coupling independent 

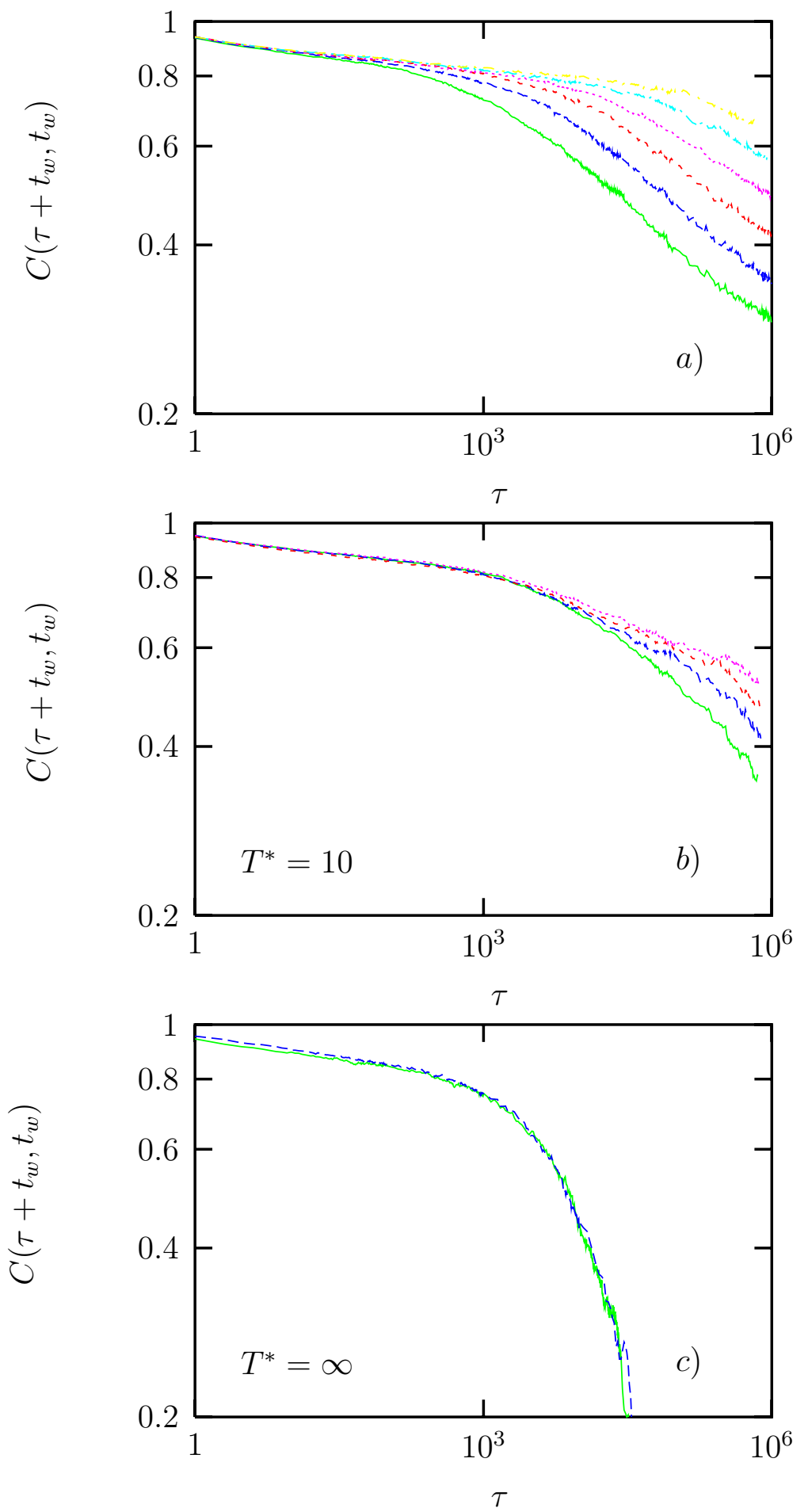

Fig. 3. Autocorrelations of 3D Edwards-Anderson model. From top to bottom: a) pure relaxtion, b) same but coupled to a weak slow bath with $T^{*}=10$, c) same but coupled to a weak slowly evolving random field (a weak slow bath with $T^{*}=\infty$ ). In b) aging has partially dissappeared, while in c) it has dissappeared completely. 
constants, on average half as strong in one system than in the other). Despite the assymmetry between the two systems (cfr. the left figure), the mutual correlations $C_{12}$ and $C_{21}$ tend to equalise more and more for longer waiting times (right figure).

\subsection{Emergence of macroscopic temperatures.}

A very encouraging feature of the effective temperatures, such as they come out in the solution of some analytic models [13], is that they remain non-zero in the limit of zero bath temperature. In other words, the temperatures stay non-zero even when Boltmann's constant is negligible. This is most welcome: if these effective temperatures are to be relevant for macroscopic cases such as colloids, sheared foams and (perhaps) granular media, they must be huge in units of the Boltmann constant, and be related to macroscopic structures.
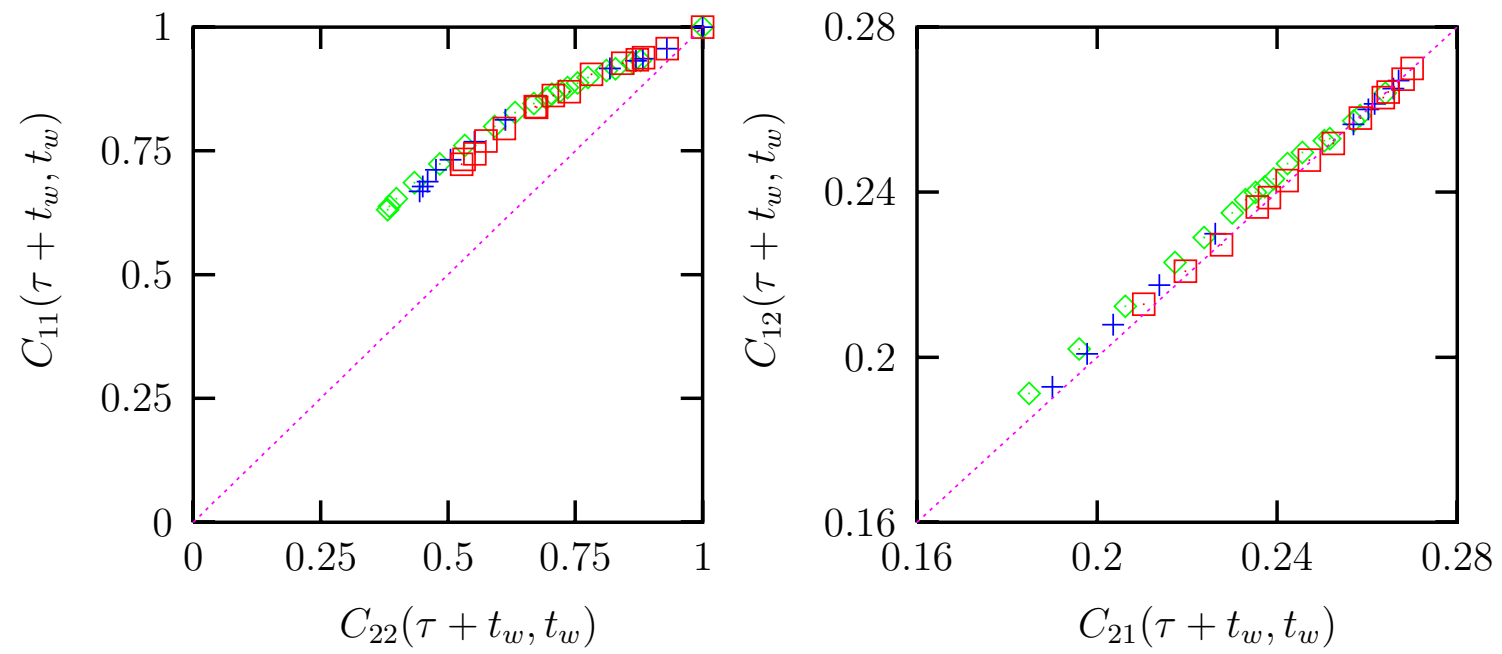

Fig. 4. Parametric plots of $C_{11}$ vs. $C_{22}$ (left) and $C_{12}$ vs. $C_{21}$ (right) for $t_{w}=3000$ (diamonds), $t_{w}=10000$ (crosses), $t_{w}=30000$ (squares). Note that the points in the right graph approach the diagonal for larger $t_{w}$.

\section{$2 \quad$ Analytical results. Symmetry breaking}

Let us outline very briefly the analytical arguments showing that this scenario 'closes'. We assume the system has Langevin dynamics:

$$
-m_{i} \ddot{\phi}_{i}-M_{i j} \phi_{j}-\frac{\partial V(\phi)}{\partial \phi_{i}}=\Gamma_{0} \dot{\phi}_{i}-\eta_{i}
$$

where $\eta_{i}$ are uncorrelated Gaussian white noises with variance $2 \Gamma_{0} T$ and $\Gamma_{0}$ is the strength of the coupling to the 'white' bath. One can also add weak 
nonconservative forces. The masses $m_{i}$ can in particular be zero.

We can encode the correlations and (causal) responses in the 'superspace' order parameter (see e.g. [14]):

$$
Q_{i j}(1,2)=C_{i j}\left(t_{1}, t_{2}\right)+\left(\bar{\theta}_{2}-\bar{\theta}_{1}\right)\left[\theta_{2} R_{i j}\left(t_{1}, t_{2}\right)-\theta_{1} R_{j i}\left(t_{2}, t_{1}\right)\right] .
$$

where $\theta_{a}, \bar{\theta}_{a}$ are Grassmann variables, and we denote the full set of coordinates in a compact form as $1=t_{1} \theta_{1} \bar{\theta}_{1}, d 1=d t d \theta d \bar{\theta}$, etc.

One can obtain a general perturbative expression for the correlations and responses ([11], see also [14]):

$\left(m_{i} \frac{\partial^{2}}{\partial t_{1}^{2}}+\Gamma_{0} D_{1}^{(2)}\right) Q_{i j}(1,2)+M_{i k} Q_{k j}(1,2)=\delta(1-2) \delta_{i j}+[\boldsymbol{\Sigma} \otimes \mathbf{Q}]_{i j}(1,2)$

where $\otimes$ stands for convolution and matrix product. The function $\Sigma$ is obtained from all diagrams with propagator $\mathbf{Q}$ with two (amputated) legs that cannot be disconnected by cutting one more line. Note that we have nowhere assumed there is quenched disorder, and that this expansion can be done for any model, independently of the dimensionality.

We now make a separation between 'fast' $\mathbf{Q}^{\mathbf{F}}$ and 'slow' $\tilde{\mathbf{Q}}$ evolution $\mathbf{Q}=\mathbf{Q}^{\mathbf{F}}+$ $\tilde{\mathbf{Q}}$. In the 'fast' regime of finite time-differences, correlations and responses can be shown [4] to satisfy FDT, and are time-translation invariant. In the SEP limit one can obtain a separate equation for the 'slow' part:

$$
M_{i k} \tilde{Q}_{k j}(1,2)-\frac{q_{k j}^{d}-q_{k j}^{E A}}{T} \tilde{\Sigma}[\tilde{Q}]_{i k}(1,2)-\frac{d_{k j}^{d}-d_{k j}^{E A}}{T} \tilde{Q}_{i k}(1,2)=\text { 'small' }
$$

Here 'small' stands for terms that vanish in the SEP limit (see below), and $\tilde{\Sigma}[\tilde{Q}]_{i k}(1,2)$ is a functional of $\tilde{Q}$ that is also obtained diagrammatically. The $q_{k j}^{d}, q_{k j}^{E A}, d_{k j}^{d}, d_{k j}^{E A}$ are parameters to be determined from (8) and the equations for $\mathrm{Q}^{\mathrm{F}}$.

\subsection{Symmetries}

In general, to the extent that one is allowed to neglect the 'small' terms in the SEP limit, Eqs. (7) are invariant with respect to any change of 'coordinates' $t_{a}$, $\theta_{a}$, and $\bar{\theta}_{a}(a=1,2, \ldots)$ with unit superjacobian [15]. This is a large symmetry group, including in particular the following: 
i. Ordinary time-reparametrizations.

$$
t_{a} \rightarrow K\left(t_{a}\right), \quad \theta_{a} \rightarrow \dot{K}\left(t_{a}\right) \theta_{a}, \quad \bar{\theta}_{a} \rightarrow \bar{\theta}_{a} .
$$

These reparametrizations were already considered by Sompolinsky and Zippelius in their seminal work [16]. Under this subgroup the order parameter Q preserves the form (6).

ii. Time-reversal.

$$
t_{a} \rightarrow-t_{a}-\beta^{*} \bar{\theta} \theta, \quad \theta_{a} \rightarrow \bar{\theta}_{a}, \quad \bar{\theta}_{a} \rightarrow \theta_{a} .
$$

These reparametrizations only preserve the form (6) if they act on a $\tilde{\mathbf{Q}}$ such that $R_{i j}\left(t-t^{\prime}\right)=\beta^{*} \partial_{t^{\prime}} C_{i j}\left(t-t^{\prime}\right) \Theta\left(t-t^{\prime}\right)$.

iii. Boson-Fermion symmetry

A group of symmetry transformations which, unlike the previous two, exchanges bosons and fermions is

$$
K\left(t_{a}\right) \rightarrow K\left(t_{a}\right)+K_{0}, \bar{\theta}_{a} \rightarrow \bar{\theta}_{a}+\bar{\epsilon}, \quad \theta_{a} \rightarrow \theta_{a}+\tilde{\epsilon}, \quad t_{a} \rightarrow t_{a}+\frac{\epsilon}{\dot{K}(t)} \bar{\theta}_{a}
$$

for any $K(t), K_{0}, \bar{\epsilon}, \tilde{\epsilon}, \epsilon$ (the latter three, odd-Grassmann parameters).

\subsection{Temperature fixing as symmetry breaking}

Let us now briefly show how this symmetry considerations prove the consistency of the ansatz. We shall for brevity only mention here the case of a single effective temperature $T^{e f f}$ for the slow dynamics.

Equation (8) (neglecting its r.h.s.) will always admit a solution invariant with respect to some residual subgroup of the group of transformations with unit superjacobian. To get a non-constant solution, we must then break (i), by choosing a time-reparametrisation $K(t)$. One can easily check that fixing an effective temperature now corresponds to breaking (iii) to a three-parameter subgroup with fixed $K(t)$ and with the restriction $\tilde{\epsilon}=T^{e f f} \epsilon$ in (11). One can further keep 'time reversal' (10), but as applied on the reparametrised time $K(t)$, and with $T^{*}=T^{e f f}$.

This may seem rather strange, but in fact it is the abstract way of describing the usual temperature fixing by a thermal bath. In any purely Hamiltonian system $\left(\Gamma_{0}=0\right)$, Eqs. (7) are invariant under the group (11) with $K(t)=t$ and four parameters $K_{0}, \epsilon, \tilde{\epsilon}$ and $\bar{\epsilon}$. If we now switch on a thermal bath of temperature $T$ (we make $\Gamma_{0}>0$ ), the group (11) is broken down by the 'bath' term proportional to $\Gamma_{0}$ to the residual three parameter subgroup [17] obtained by restricting $\tilde{\epsilon}=T \epsilon$. 


\subsection{Sensitivity and the matching problem}

The neglect of the 'small' terms in the right hand side of equation (8) brings about the invariance with respect to super-reparametrisations. However, the true equations do not have so many symmetries. In order to see what is the situation, we note that equation (8) is of the form:

$$
\text { [ (Super) Reparametrization Invariant ] = 'small', non-invariant }
$$

If we find one solution to this equation by setting the l.h.s. to zero, any super-reparametrization is also a solution. If the solution to l.h.s. $=0$ breaks reparametrization invariance, one obtains thus an infinite number of solutions. In order to fix the correct one, one must take into account the 'small' terms in the r.h.s. This requires a calculation going beyond the zero entropy production limit (small but finite forcing or long but finite waiting times), and this is a much harder task, indeed a problem that remains to be solved.

On the other hand, apart from being a technical nuisance, this has a physical meaning. Because terms that are arbitrarily small decide which reparametrization is the good one, systems having non-trivial solutions to (8) are sensitive to perturbations that are arbitrarily weak, a general feature of systems with slow dynamics.

\section{Perspectives}

Given the number of not entirely obvious relations this scenario proposes, the least one can now say is that it has heuristic value, even if the fact of having been inspired by mean-field spin-glass theory is sometimes viewed as an original sin. There has been recently quite a lot of numerical activity to test the predictions, with encouraging results. The experimental exploration of structural glasses from this point of view is also now beginning.

The solution is consistent in general, since it corresponds to the breaking of a symmetry that is a general property of slow evolution down to a residual subgroup (having the physical meaning of partial thermalisations). Just as in any partial symmetry breaking situation, one cannot with such arguments alone assure that in some model this symmetry will not be broken further, or in a different way. One may perhaps hope that real proofs concerning the generic situation in the SEP limit of extended systems will be one day available. 


\section{References}

[1] This point of view has been advocated by Horner [2]. See also [3].

[2] H. Horner, Z. Phys. B57 (1984) 29; ibid. (1984) 39.

[3] L. F. Cugliandolo, J. Kurchan, P. Le Doussal and L. Peliti; Phys. Rev. Lett. 78 (1997), 350.

[4] L. F. Cugliandolo, D.S. Dean and J. Kurchan; Phys. Rev. Lett. 79 (1997) 2168.

[5] R. Kubo, M. Toda and N. Hashitume; Statistical Physics II. Nonequilibrium Statistical Mechanics, Springer-Verlag, 1992;

S. R. De Groot and P. Mazur; Non-equilibrium thermodynamics, Dover Pub., New York, 1984.

[6] E. Marinari, G. Parisi, F. Ricci-Tersenghi and J.J. Ruiz-Lorenzo; J. Phys. A 31 (1998) 2611.

[7] G. Parisi, Phys. Rev. Lett. 79 (1997) 3660.

[8] J-L Barrat and W. Kob, cond-mat/9806027.

[9] A. Barrat, Phys. Rev. E57 (1998) 3629.

[10] L. F. Cugliandolo, J. Kurchan and L. Peliti; Phys. Rev. E55 (1997) 3898.

[11] L.F. Cugliandolo and J. Kurchan, 'Thermal properties of systems with slow dynamics', in preparation.

[12] This is much in the spirit of: G. Parisi and M.A. Virasoro J. Physique (Paris) 50 (1986) 3317, and R. Monasson, Phys. Rev. Lett. 752847 (1995).

[13] J-P Bouchaud, L. F. Cugliandolo, J. Kurchan and M. Mézard; condmat/9702070; Spin-glasses and random fields, A. P. Young ed. (World Scientific, Singapore 1998).

[14] J.P. Bouchaud, L.F. Cugliandolo, J. Kurchan et M. Mézard. Physica A226 (1996) 243.

[15] S. Franz and J. Kurchan. Europhys. Lett. 20 (1992) 197.

[16] H. Sompolinsky, Phys. Rev. Lett. 47 (1981) 935; H. Sompolinsky and A. Zippelius, Phys. Rev. B25 (1982) 6860.

[17] E. Gozzi; Phys.Rev. D30 (1984) 1218. 